\title{
All-cause mortality rates in patients with type 1 diabetes mellitus compared with a non-diabetic population from the UK general practice research database, 1992-1999
}

Received: 27 April 2005 / Accepted: 31 October 2005 / Published online: 24 January 2006

C) Springer-Verlag 2006

\begin{abstract}
Aims/hypothesis: We compiled up to date estimates of the absolute and relative risk of all-cause mortality in patients with type 1 diabetes in the UK. Materials and methods: We selected patients with type 1 diabetes $(n=7,713)$, and for each of these diabetic subjects five age- and sex-matched control subjects without diabetes $(n=38,518)$ from the General Practice Research Database (GPRD). Baseline was 1 January 1992; subjects were followed until 1999. The GPRD is a large primarycare database containing morbidity and mortality data of a large sample representative of the UK population. Deaths occurring in the follow-up period were identified. Results: The study comprised 208,178 person-years of follow-up. The prevalence of type 1 diabetes was 2.15/1,000 subjects in 1992 (mean age 33 years, SD 15). Annual mortality rates were 8.0 per 1,000 person-years (95\% CI 7.2-8.9) in type 1 diabetic subjects compared with 2.4 per 1,000 person-years $(95 \%$ CI $2.2-2.6)$ in those without diabetes (hazard ratio [HR] $=3.7,95 \%$ CI 3.2-4.3).
\end{abstract}

S. S. Soedamah-Muthu · J. H. Fuller · H. M. Colhoun Department of Epidemiology and Public Health, Royal Free and University College London Medical School, London, UK

S. S. Soedamah-Muthu $(\bowtie)$

Julius Center for Health Sciences and Primary Care,

University Medical Centre Utrecht,

Huispostnr str. 6.131,

P.O. Box 85500, 3508 GA, Utrecht, The Netherlands,

e-mail: s.s.soedamah-muthu@umcutrecht.nl

Tel.: +31-30-2509379

Fax: $+31-30-2505485$

H. E. Mulnier · V. S. Raleigh · R. A. Lawrenson Postgraduate Medical School, University of Surrey, Guildford, Surrey, UK

V. S. Raleigh

Healthcare Commission, Finsbury Tower,

London, UK

H. M. Colhoun

Conway Institute of Biomolecular and Biomedical Research, University College Dublin,

Dublin, Ireland
The increased mortality rates in patients with type 1 diabetes were apparent across all age-bands. The HR was higher in women $(\mathrm{HR}=4.5,95 \%$ CI 3.5-5.6 compared with non-diabetic women) than men ( $\mathrm{HR}=3.3,95 \%$ CI 2.7-4.0), such that the sex difference $(p<0.0001)$ in mortality in the non-diabetic population was abolished $(p=0.3)$ in the type 1 diabetic patients. The predominant cause of death in patients with type 1 diabetes was cardiovascular disease. Conclusions/interpretation: Despite advances in care, UK mortality rates in the past decade continue to be much greater in patients with type 1 diabetes than in those without diabetes.

Keywords All-cause mortality - General practice General Practice Research Database - Type 1 diabetes mellitus

Abbreviations CVD: cardiovascular disease - GP: general practitioner - GPRD: General Practice Research Database - HR: hazard ratio - MI: myocardial infarction . OXMIS: Oxford Medical Information System - PPA: Prescription Pricing Authority - SMR: standardised mortality ratio

\section{Introduction}

Type 1 diabetes is associated with an increased risk of allcause mortality compared with the general population. The magnitude of this risk has not been estimated very precisely. Many previous studies estimating relative risks for mortality in individuals with type 1 diabetes were small (number of patients with type 1 diabetes varied between 241 and 3,228) resulting in a wide range of estimates of relative risk for mortality (2- to 15 -fold). General population rates were used for comparison, which contain those with diabetes, causing underestimation of the true relative risk [1-10].

The relative risks of mortality associated with type 1 diabetes vary widely between different countries $[9,11]$. In the WHO Multinational Study of Vascular Disease in 
Diabetes [9] relative risks of all-cause mortality were estimated in patients with type 1 and type 2 diabetes from ten centres throughout the world, followed for about 10 years. Standardised mortality ratios (SMRs) of, respectively, 188 (95\% CI 113-294) and 338 (95\% CI 214$507)$ were found, for example, in men and women with type 1 diabetes from the London cohort and SMRs of, respectively, 682 (95\% CI 495-915) and 655 (95\% CI 435946) were found in men and women with type 1 diabetes from the Berlin centre, all compared with the general population. Similarly, the Diabetes Epidemiology Research International Mortality Study Group reported a variation in relative risks of all-cause mortality between Japanese and Finnish patients with type 1 diabetes diagnosed from 1965 to 1979 and followed until 1994 [11]. This diversity in relative risks of mortality between countries illustrates the importance of having mortality estimates specific for the UK. In the UK, the Diabetes UK cohort investigators reported SMRs in men and women of 2.7 (95\% CI 2.5-2.9) and 4.0 (95\% CI 3.6-4.4), respectively [12]. This analysis included 23,752 patients whose diabetes mellitus was treated with insulin and who were younger than 30 years. These patients were drawn from a large Diabetes UK register and several smaller registers with notifications from physicians, hospital admissions, general practitioners (GPs), pharmacists and specialist nurses, from England, Wales, Scotland and Northern Ireland, followed up to 1997 [12]. The investigators compared total mortality rates in their diabetes population indirectly with the UK general population, which also included subjects with diabetes, and did not formally evaluate insulin therapy. It is not clear to what extent recent advances in diabetes care have reduced the excess mortality in patients with diabetes. To provide up to date precise estimates of the relative risk of mortality in patients with type 1 diabetes in the UK, we used data from the General Practice Research Database (GPRD). The GPRD contains more than 35 million patient-years of data and is an ideal source of primary-care data to make direct comparisons with a non-diabetic group. It provides a unique opportunity to estimate morbidity and mortality rates from a large cohort of patients with diabetes who have already contributed many years of follow-up from a large number of general practices. Excellent prescribing data can be obtained from this database, because GPs use their practice system to generate prescriptions. Insulin dependency can then be evaluated in patients with diabetes. The aim of this study is, therefore, to estimate absolute and relative all-cause mortality rates in patients with type 1 diabetes compared with an age and sex matched nondiabetic comparison group from the GPRD over a study period from 1992 to 1999 .

\section{Subjects, materials and methods}

\section{Study design}

This was a cohort study of type 1 diabetes and a comparison group without diabetes selected from the GPRD. The method for selecting practices and the validation of the data have been described elsewhere [13-16]. The GPRD was set up in 1987 and comprises data from a large network of participating general practices that undertake to enter a minimum set of information in a standardised way onto a pre-specified database for all their patients. Participating practices are trained to record medical information in a standard manner.

The minimum dataset that practices have to record is all significant current and past medical conditions, including all hospitalisations, pregnancies, childbirths and deaths and all prescriptions issued. The majority of prescriptions are issued directly from the database, so the data are therefore comprehensive. Prescribed drugs are coded on the database with the Prescription Pricing Authority (PPA) codes. The coding system used for diagnoses and symptoms is a modification of the Oxford Medical Information System (OXMIS) classification and READ codes (adopted by the UK Department of Health for use in general practice). Unlike International Classification of Disease-10, OXMIS/ READ codes are continually updated. Therefore in capturing any set of diagnoses, e.g. diabetes, from the GPRD the researcher must first conduct a wide capture of all possible relevant codes consistent with the diagnosis that are then manually verified as being relevant. A number of studies have confirmed the validity of the diagnostic and prescribing data contained in the GPRD ( $>80 \%$ agreement) $[15,17-20]$. Ethical approval was given by the GPRD Scientific and Ethical Advisory Group.

\section{Diabetes population}

At baseline, 1 January 1992, the GPRD contained information on 3,595,966 patients from 603 general practices across England, Wales, Scotland and Northern Ireland. The age and sex distributions of the population included in the GPRD are similar to the Office for National Statistics estimates for the total population in England and Wales (1992) [16].

All subjects with at least 6 months' data prior to 1 January 1992 were considered eligible for inclusion. The final censoring date was 1 October 1999. To select all of those with diabetes from the GPRD, we first defined a current set of all possible diabetes-related OXMIS/READ/ PPA codes from the database. The list of codes was checked by four investigators and all irrelevant codes were omitted (e.g. bronze diabetes, diabetes insipidus, child with diabetes etc.). The records of all subjects on the database with any diabetes-related codes were then extracted from the database. This yielded 64,640 potential patients with diabetes mellitus who were then classified into type 1 diabetes using the following algorithm: all patients currently on insulin who were aged $\leq 35$ years at treatment with insulin, or aged $\leq 35$ years at diagnosis of diabetes. If oral hypoglycaemic drugs were prescribed, the duration for inclusion as type 1 diabetes was restricted to $\leq 1.5$ years. Of 7,713 subjects thus defined as having type 1 diabetes, $3 \%$ (224) had used oral hypoglycaemic drugs for less than 
1.5 years in addition to insulin. A random sample of the type 1 diabetic patients was checked to ensure the validity of the algorithm, by manually checking the entire record of the patients against the algorithm. Newly registered or newly diagnosed patients with type 1 diabetes occurring between 1992 and 1999 were not included.

\section{Non-diabetic subjects}

The non-diabetic comparison group was a random sample of those without any OXMIS/READ/PPA code indicating diabetes. For each diabetic patient, five comparison subjects matched for age and sex were selected. The matching variables used were year of birth and sex. A comparison was also made with an age-, sex- and practice-matched comparison group and since the results were similar, only the ageand sex-matched data will be presented.

\section{Capture and classification of deaths}

The following strategy was used to identify and classify deaths. When a patient dies the GP usually records the death using an OXMIS/READ code. A list of all possible codes that might indicate death was made, e.g. 'on examination dead', 'fatal myocardial infarction'. The list of codes was then applied to the database to identify all patients with these codes in the type 1 diabetes and comparison cohorts. As well as having a code suggesting they have died, patients are usually deregistered from the database, so the registration status was also extracted. Ninety percent of potential deaths had both death-related codes as well as a deregistration status confirming the death, allowing us to reliably assess when a patient had died. Where the medical record contained only a code for death, but the patient had not been deregistered $(10 \%)$, subjects were included only if their medical record ceased on the same day as the death code was recorded. Very occasionally a death code was identified but the medical record continued. These patients were not classified as dead. We finally classified 807 deaths as being definite, which were used for the main analyses.

To determine the cause of death, a list of all diagnostic/ symptom codes that were entered on the same date as the code associated with death was compiled for all the 807 subjects. Cause of death was assigned by two investigators scanning through this list: $65 \%(523 / 807)$ of deaths had a single cause of death, $22 \%(175 / 807)$ had two causes of death, 9\% (75/807) had three causes of death and 4\% $(34 / 807)$ had four or more possible causes of death. For those with two or more codes for potential causes of death, in general the cause of death could be assigned unequivocally, e.g. myocardial infarction (MI), ischaemic heart disease and death. We established in advance a standard set of rules for assigning cause of death that were consistently applied so that any misclassification should be independent of diabetes status. For example, cancer superseded all other causes of death, then MIs and then other causes. A grouping was made into broader categories, for example, ischaemic heart disease, ischaemia myocardial, heart attack, ventricular fibrillation, and coronary thrombosis or coronary artery atheroma were all classified as 'CHD death'.

\section{Statistical analyses}

The statistical package STATA (STATA 7.0; Stata, College Station, TX, USA) was used to perform all statistical analyses. A $p$ value $<0.05$ was considered to be statistically significant. Duration of diabetes was calculated up to 1 January 1992 using the first date of diagnosis of diabetes. In $4 \%$ of all subjects duration of diabetes was uncertain.

Subjects contributed either until their death, or until they left the GPRD or until the censoring date of 1 October 1999, whichever occurred first. Reasons for leaving the GPRD were: (1) patient left the practice; or (2) the practice left the database. Crude absolute annual mortality rates and 95\% CIs were calculated for patients with type 1 diabetes and those without, for men and women separately, per 1,000 person-years.

Cox proportional hazards models were used to calculate crude risk ratios or hazard ratios (HRs) (and 95\% CIs) for all-cause mortality in patients with type 1 diabetes compared with non-diabetic comparison subjects. We used subjects' attained or current age as the underlying time scale in the Cox proportional hazards models (stset ageout, fail[death=1] enter[again]). Mortality rates and relative mortality risk (HRs) were calculated in those with type 1 diabetes compared with those without diabetes in each age-band (stsplit command in STATA) and by sex. To obtain mortality rates per 1,000 person-years we used the strate command in STATA as follows: strate case age-band sex, per(1,000). To obtain HRs for each age and sex category we used for example: stcox case if age-band==1 $\& \operatorname{sex}==1$. Matching was taken into account in the Cox proportional hazards models by estimating HRs associated with diabetes in a model stratified by the matching variables (year of birth and sex). Cause-specific relative mortality risks were also calculated. Nelson-Aalen cumulative hazards estimates were plotted by current age for men and women with type 1 diabetes and non-diabetic comparison subjects. The proportional hazards assumption was assessed by observing whether the lines were parallel for any comparison using 'log-log plots'. (with log-log survival probabilities on the $y$-axis and log current age on the $x$-axis. The Schoenfeld method (with scaled Schoenfeld residuals) was used to test for departure from the assumption that for type 1 diabetes vs those without diabetes the HR was constant throughout the period of follow up [21]. The hazards were not proportional when comparing men and women, for those with type 1 diabetes and those without. Therefore, the data were shown for men and women separately. Accordingly, all analyses are stratified by sex.

Interaction terms were fitted to assess the interaction of sex and diabetes to test whether the effect of type 1 diabetes 
vs no diabetes on mortality was constant for men and women. Likelihood ratio tests were carried out, comparing the value of the maximum likelihood of the models with and without the interaction term.

The effect of duration of diabetes on mortality risk among those with type 1 diabetes was also assessed using current age as the underlying time scale in the Cox proportional hazards models.

\section{Results}

Description of the study population

The prevalence of type 1 diabetes in 1992 was 2.15/1,000 subjects $(7,713 / 3,595,966$ subjects). A description of the total population is given in Table 1 . The mean age of the cohort in 1992 was 33 years (SD 15) and ranged from age 1 to 96 . Patients with type 1 diabetes died relatively younger (mean age 55) than non-diabetic comparison subjects (mean age 62). The mean observation period per person was 4.50 years (SD 2.26) and ranged from 0.003 to 7.7 years. The 7,713 patients with type 1 diabetes and 38,518 comparison subjects contributed a total of 208,284 person-years of follow-up.

\section{Total mortality}

Annual mortality rates were 8.0 (95\% CI 7.2-8.9) in those with type 1 diabetes and 2.4 (95\% CI 2.2-2.6) per 1,000 person-years in comparison subjects. Increased mortality rates (per 1,000 person-years) were found in men $(8.4,95 \%$ CI 7.3-9.7) and women (7.4, 95\% CI 6.3-8.8) with type 1 diabetes compared with men $(2.8,95 \%$ CI $2.5-3.1)$ and women $(1.9,95 \%$ CI 1.6-2.2) without diabetes. The HR was $4.5(95 \%$ CI $3.5-5.6)$ in diabetic vs non-diabetic women and was $3.3(95 \%$ CI 2.7-4.0) in diabetic vs nondiabetic men, after stratification by year of birth and sex.

Table 1 Description of population

\begin{tabular}{lll}
\hline & $\begin{array}{l}\text { Type 1 } \\
\text { diabetes } \\
(n=7,713)\end{array}$ & $\begin{array}{l}\text { Comparison } \\
\text { group } \\
\text { without diabetes } \\
(n=38,518)\end{array}$ \\
\hline $\begin{array}{l}\text { Proportion men }(n) \\
\begin{array}{l}\text { Mean age at first diagnosis of } \\
\text { diabetes in years (SD) }\end{array}\end{array}$ & $\begin{array}{l}55 \%(4,216) \\
18.5(9.1)\end{array}$ & - \\
$\begin{array}{l}\text { Mean age at baseline } \\
\text { in years (SD) }\end{array}$ & $33(21,059)$ & $33(15)$ \\
$\begin{array}{l}\text { Mean age at death in } \\
\text { years (SD) }\end{array}$ & $55(16)$ & $62(17)$ \\
$\begin{array}{l}\text { Mean duration of } \\
\text { diabetes in years (SD) }\end{array}$ & $15(12)$ & - \\
\hline
\end{tabular}

For the total population, a 3.7 -fold (95\% CI 3.2-4.3) increased risk was found in those with type 1 diabetes mellitus compared with those without, after stratification by year of birth and sex.

The mortality rates were significantly higher in patients with type 1 diabetes than comparison subjects across all age-bands (Table 2). Analyses presented in Table 2 used attained or current age as the underlying time scale. In comparison with analyses using baseline age as the underlying time scale, these did not alter the results, in particularly not for the HRs. The HR for type 1 diabetic patients vs those without diabetes aged $\leq 35$ years was 4.9 (95\% CI 2.8-8.4) for men and 6.9 (95\% CI 3.2-15.0) for women using attained age, compared with 4.6 (95\% CI 2.9-7.3) for men and 8.7 (95\% CI 4.4-17.2) for women using baseline age. The highest HRs were shown for the youngest subjects (age at baseline <35), gradually decreasing in the elderly patients with type 1 diabetes. For women, these HRs were slightly but not significantly ( $p$ values for sex by diabetes interaction split by age-bands were all greater than 0.05) higher for all ages, up to about a 12-fold increased risk in the youngest subjects and a 2-fold increased risk in the older groups.

The Nelson-Aalen cumulative hazards function illustrates the increased mortality risk in patients with type 1 diabetes compared with controls with current age for both men and women (Fig. 1). The overall relative mortality risk was higher in men than in women for subjects without diabetes $(\mathrm{HR}=1.5,95 \%$ CI $1.3-1.9$ for men vs women, $p<0.0001)$. In type 1 diabetic patients, on the other hand, the difference in the HR of mortality in men compared with women was not significant $(\mathrm{HR}=1.1,95 \%$ CI $0.9-1.4$, $p=0.3$ ). There was evidence that the effect of diabetes vs no diabetes on mortality was not constant between men and women (fitting interaction of sex with diabetes, likelihood ratio test, $p=0.02$ ).

\section{Cause-specific mortality}

The HRs for fatal cardiovascular disease (CVD) $(n=167$, defined as MI death, acute CHD death and cerebrovascular accident death), cancer deaths $(n=201)$ and mortality from other causes were $(n=439)$ were calculated. The HR for fatal CVD was significantly raised in patients with type 1 diabetes compared with non-diabetic subjects $(\mathrm{HR}=7.4$, 95\% CI 5.4-10.1), with higher HRs in women ( $\mathrm{HR}=11.6$, 95\% CI 6.7-20.1) than in men (HR=5.8, 95\% CI 3.9-8.6), but no significant diabetes-sex interaction $(p=0.07)$. For cancer deaths, the HR of cancer mortality did not significantly differ between the two groups $(\mathrm{HR}=1.05$, $95 \%$ CI $0.72-1.52$ ), with similar findings when the results were split by sex. For mortality from other causes, also significantly raised HRs were found of 4.5 (95\% CI $3.7-$ 5.4 ) in those with type 1 diabetes compared with those without diabetes. 
Table 2 Mortality rates (MRs) and relative risk (hazard ratios [HRs]) of all-cause mortality in patients with type 1 diabetes compared with non-diabetic comparison group stratified by (current) age-bands

\begin{tabular}{|c|c|c|c|c|c|c|c|c|}
\hline \multirow{3}{*}{$\begin{array}{l}\text { Age- } \\
\text { band } \\
\text { (years) }\end{array}$} & \multirow{3}{*}{$\begin{array}{l}\text { Person- } \\
\text { years }\end{array}$} & \multirow{3}{*}{$\begin{array}{l}\text { Number } \\
\text { of deaths }\end{array}$} & \multicolumn{3}{|l|}{ Men } & \multicolumn{3}{|l|}{ Women } \\
\hline & & & \multicolumn{2}{|c|}{$\mathrm{MR} / 1,000$ patient-years $(95 \% \mathrm{CI})$} & \multirow{2}{*}{$\begin{array}{l}\text { Diabetes vs } \\
\text { comparison } \\
\text { group, HR } \\
(95 \% \mathrm{CI})\end{array}$} & \multicolumn{2}{|c|}{$\mathrm{MR} / 1,000$ patient-years $(95 \% \mathrm{CI})$} & \multirow{2}{*}{$\begin{array}{l}\text { Diabetes vs } \\
\text { comparison } \\
\text { group, HR } \\
(95 \% \mathrm{CI})\end{array}$} \\
\hline & & & Diabetes & $\begin{array}{l}\text { Without } \\
\text { diabetes }\end{array}$ & & Diabetes & $\begin{array}{l}\text { Without } \\
\text { diabetes }\end{array}$ & \\
\hline$\leq 35$ & 100,538 & 75 & $2.4(1.6-3.5)$ & $0.5(0.3-0.7)$ & $4.9(2.8-8.4)$ & $1.7(1.0-2.7)$ & $0.2(0.1-0.4)$ & $6.9(3.2-15.0)$ \\
\hline $36-45$ & 51,417 & 84 & $5.0(3.5-7.2)$ & $1.0(0.7-1.4)$ & $5.2(3.1-8.8)$ & $4.3(2.7-6.8)$ & $0.4(0.2-0.7)$ & $11.8(4.9-28.3)$ \\
\hline $46-55$ & 31,350 & 133 & $12.0(8.8-16.4)$ & $1.9(1.4-2.7)$ & $6.5(4.1-10.4)$ & $10.0(6.8-14.6)$ & $2.7(2.0-3.8)$ & $3.7(2.2-6.1)$ \\
\hline $56-65$ & 15,841 & 186 & $28.3(21.1-37.9)$ & $8.4(6.7-10.7)$ & $3.4(2.3-5.0)$ & $28.6(20.6-39.6)$ & $6.2(4.6-8.5)$ & $4.5(2.9-7.1)$ \\
\hline $66-75$ & 7,181 & 176 & $42.4(29.5-61.0)$ & $22.4(18.0-27.9)$ & $1.9(1.2-2.9)$ & $44.1(29.8-65.3)$ & $11.6(8.4-16.0)$ & $3.8(2.3-6.3)$ \\
\hline$\geq 76$ & 1,957 & 153 & $113.2(73.0-175.5)$ & $77.7(62.0-97.2)$ & $1.6(0.96-2.8)$ & $136.6(83.7-223.0)$ & $46.4(34.1-63.3)$ & $3.1(1.6-5.9)$ \\
\hline Total & & 807 & & & & & & \\
\hline
\end{tabular}

All HRs are stratified by year of birth and sex

Other analyses

The inclusion of all type 1 diabetic patients on insulin, without exclusion of those using oral hypoglycaemic agents, did not alter the results. Among those with diabetes, diabetes duration was not significantly related to mortality risk independently of age, with which it was highly correlated (HR $=1.01,95 \%$ CI $1.00-1.02)$ for every 1 year increment in duration in the Cox proportional hazards models.

\section{Conclusions}

This study evaluated absolute and relative mortality rates in patients with type 1 diabetes directly compared with a nondiabetic comparison group, using a validated algorithm to define type 1 diabetes. The analysis shows clearly that, despite advances in the care of patients with diabetes, mortality rates continue to be greatly elevated in those with type 1 diabetes in the UK. Furthermore, the greater elevation in risk in women than men with diabetes compared

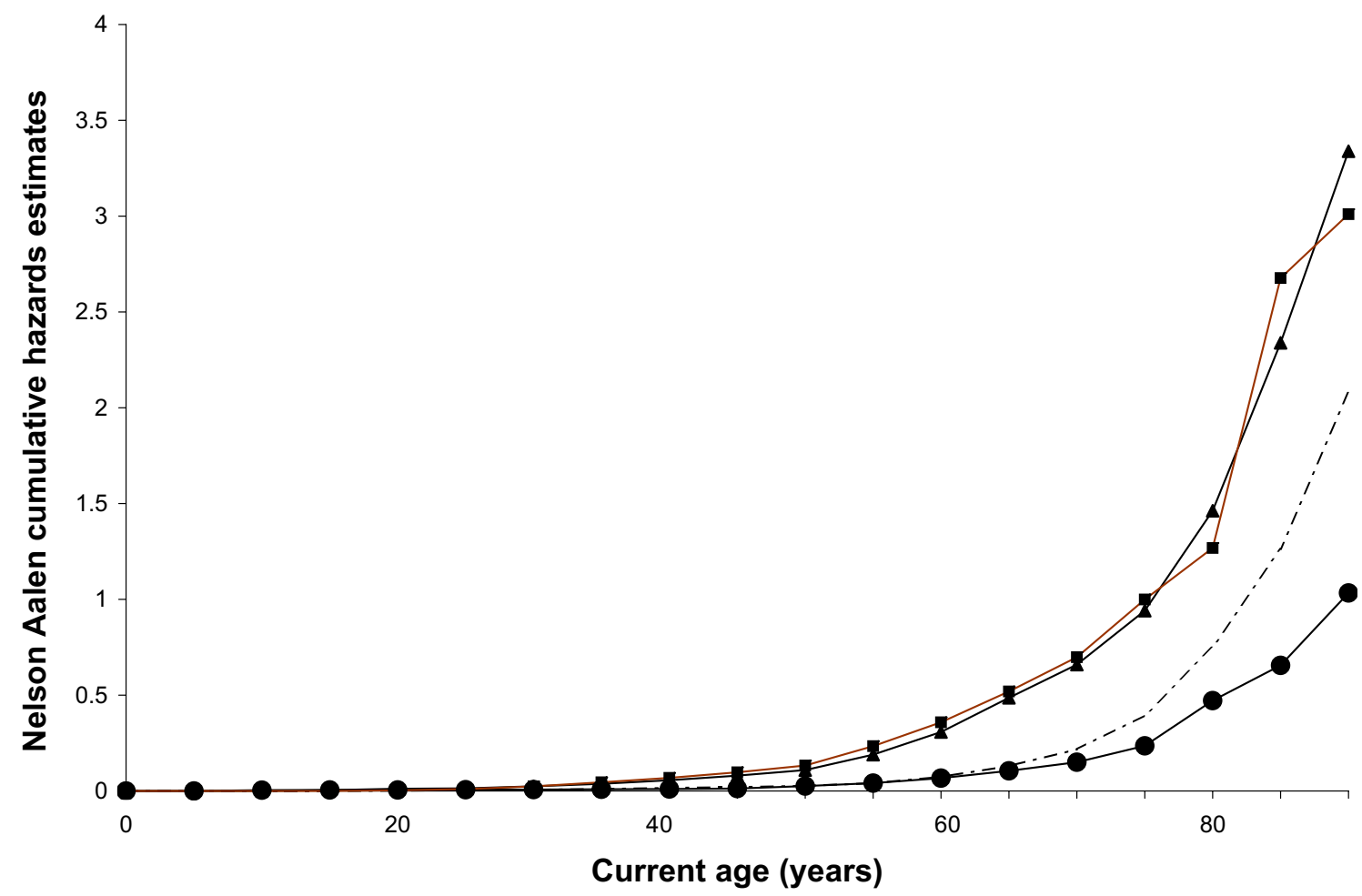

Fig. 1 Nelson-Aalen cumulative hazards functions for total mortality by current age, in men (squares) and women (triangles) with type 1 diabetes, compared with nondiabetic men (dotted dashed line) and women (circles) 
with the general population has persisted. The main cause of death in patients with type 1 diabetes was CVD, with greatly elevated relative risks compared with those without diabetes. This large dataset means that our mortality estimates are precise, and have narrow CIs. Clearly a great deal remains to be done to improve our understanding of the causes and prevention of complications of diabetes.

For external validation, the prevalence of type 1 diabetes as well as the mortality rates in the non-diabetic control group were compared with other national and international sources. The prevalence of type 1 diabetes was $2.15 / 1,000$ persons in 1992 in the GPRD, which is comparable with the prevalence rates reported by the International Diabetes Federation in 2000 for the UK, France, Germany and the Netherlands, respectively, of 3.4, 1.6, 1.0 and 2.6 per 1,000 persons (http://www.idf.org data accessed on 17 September 2003). In addition, similar (absolute) age-specific death rates were found for the nondiabetic control group in the GPRD in comparison with the Office for National Statistics 2001.

Our results were similar to the results of the large Diabetes UK cohort study [12]. They found standardised mortality rates of, respectively, 4.0 (95\% CI 3.6-4.4) and 2.7 (95\% CI 2.5-2.9) in women and men, using data up to 1997. Our more recent data showed relative risks of 4.5 (95\% CI 3.5-5.6) in women and 3.3 (95\% CI 2.7-4.0) in men. This is a robust finding based on the two largest studies in the UK. Other much smaller studies in Europe and the United States estimated standardised mortality rates ranging from 2- to 15-fold [1-10].

Increased mortality risks were found across all agebands, but these were especially high in the younger patients with type 1 diabetes compared with subjects without diabetes, and especially in young women. Although we did not find a significantly higher risk ratio in women compared with men with type 1 diabetes vs those without, the sex difference was abolished in those with diabetes $(p=0.3)$. This greater risk of mortality in young women with type 1 diabetes has been reported before [6, 12], but remains poorly understood. With respect to causespecific mortality, diabetes was associated with an increased HR for CVD deaths and other miscellaneous causes of death, but not cancer deaths.

A limitation of our study is that risk factor data are not complete in the database, and being complex to extract, we therefore did not carry out proper risk stratification or construct a much needed risk engine for identifying those patients with type 1 diabetes at greatest risk of specific causes of death. Although many GPs do report risk factor data, they were not required at the setup to provide this information. Furthermore, no centralised laboratories have been used, for example for the measurement of glycated haemoglobin. The care given by GPs to patients with type 1 diabetes, is however, typical of care received by these patients in general.

The main strengths of the use of GPRD data are the large sample size, the longitudinal design, the use of unselected typical diabetes encountered in general practice, the excellent prescribing data and the precise estimates of mortality and major clinical events. A matched nondiabetic reference group provides an ongoing direct comparison group matched for population characteristics as well as providing a validation sample for quantifying how the event rate in the study population compares with the UK as well as with other countries. Finally, there are a number of data quality checks in place to check consultations and prescriptions, as well as the completeness of demographic and registration data. Furthermore, several validation studies $[15,17-20]$ have found high agreement rates $(>80 \%)$.

Few studies using GPRD data have selected patients with diabetes mellitus [22-25], and most of these used only diabetes-related OXMIS/READ codes and no treatment information to select patients with diabetes [23-25]. For the first time, a validated algorithm was used to select those with type 1 diabetes mellitus.

The main conclusion is that mortality rates continue to be greatly elevated in patients with type 1 diabetes in the UK, despite improvements in diabetes care. The major cause of death is CVD in patients with type 1 diabetes. Forthcoming studies will evaluate cause-specific mortality rates in more detail. The precise estimates found in this study will allow comparison of mortality rates in the UK and with other countries.

Acknowledgements We would like to thank all participating GPs and study participants. This study was supported by a grant from the British Heart Foundation.

\section{References}

1. Dorman JS, LaPorte RE, Kuller LH et al (1984) The Pittsburgh insulin-dependent diabetes mellitus (IDDM) morbidity and mortality study. Mortality results. Diabetes 33:271-276

2. Moss SE, Klein R, Klein BE (1991) Cause-specific mortality in a population-based study of diabetes. Am J Public Health 81:11581162

3. Sartor G, Nystrom L, Dahlquist G (1991) The Swedish Childhood Diabetes Study: a seven-fold decrease in shortterm mortality? Diabet Med 8:18-21

4. Joner G, Patrick S (1991) The mortality of children with type 1 (insulin-dependent) diabetes mellitus in Norway, 1973-1988. Diabetologia 34:29-32

5. Walters DP, Gatling W, Houston AC et al (1994) Mortality in diabetic subjects: an eleven-year follow-up of a communitybased population. Diabet Med 11:968-973

6. Swerdlow AJ, Jones ME (1996) Mortality during 25 years of follow-up of a cohort with diabetes. Int J Epidemiol 25:1250-1261

7. Warner DP, McKinney PA, Law GR, Bodansky HJ (1998) Mortality and diabetes from a population based register in Yorkshire 1978-93. Arch Dis Child 78:435-438

8. Nishimura R, LaPorte RE, Dorman JS et al (2001) Mortality trends in type 1 diabetes. The Allegheny County (Pennsylvania) Registry 1965-1999. Diabetes Care 24:823-827

9. Morrish NJ, Wang SL, Stevens LK, Fuller JH, Keen H (2001) Mortality and causes of death in the WHO multinational study of vascular disease in diabetes. Diabetologia 44 (Suppl 2):S14S21

10. Wibell L, Nystrom L, Ostman J et al (2001) Increased mortality in diabetes during the first 10 years of the disease. A population-based study (DISS) in Swedish adults 15-34 years old at diagnosis. J Intern Med 249:263-270 
11. Asao K, Sarti C, Forsen T et al (2003) Long-term mortality in nationwide cohorts of childhood-onset type 1 diabetes in Japan and Finland. Diabetes Care 26:2037-2042

12. Laing SP, Swerdlow AJ, Slater SD et al (1999) The British Diabetic Association Cohort Study, I: all-cause mortality in patients with insulin-treated diabetes mellitus. Diabet Med 16:459-465

13. Walley T, Mantgani A (1997) The UK General Practice Research Database. Lancet 350:1097-1099

14. Garcia Rodriguez LA, Perez GS (1998) Use of the UK general practice research database for pharmacoepidemiology. Br J Clin Pharmacol 45:419-425

15. Jick H, Terris BZ, Derby LE, Jick SS (1992) Further validation of information recorded on general practitioner based computerised data resources in the United Kingdom. Pharmacoepidemiol Drug Saf 1:349

16. Anonymous (1996) The general practice research database: information for researchers. Office for National Statistics, London, pp 1-26

17. Nazareth I, King M, Haines A, Rangel L, Myers S (1993) Accuracy of diagnosis of psychosis on general practice computer system. BMJ 307:32-34
18. Van Staa T-P, Abenhaim L (1994) The quality of information recorded on a UK database of primary care records: a study of hospitalizations due to hypoglycemia and other conditions. Pharmacoepidemiol Drug Saf 3:15-21

19. Jick H, Jick SS, Derby LE (1991) Validation of information recorded on general practitioner based computerised data resource in the United Kingdom. BMJ 302:766-768

20. Hollowell J (1997) The General Practice Research Database: quality of morbidity data. Popul Trends 36-40

21. Schoenfeld D (1982) Partial residuals for the proportional hazards regression model. Biometrika 69:239-241

22. Jick SS, Stender M, Myers MW (1999) Frequency of liver disease in type 2 diabetic patients treated with oral antidiabetic agents. Diabetes Care 22:2067-2071

23. Ruigomez A, Garcia Rodriguez LA (1998) Presence of diabetes related complication at the time of NIDDM diagnosis: an important prognostic factor. Eur J Epidemiol 14:439-445

24. Kornegay CJ, Vasilakis-Scaramozza C, Jick H (2002) Incident diabetes associated with antipsychotic use in the United Kingdom general practice research database. J Clin Psychiatry 63:758-762.

25. Wood SL, Jick H, Sauve R (2003) The risk of stillbirth in pregnancies before and after the onset of diabetes. Diabet Med 20:703-707 